Alexandra Milligan, Chris Mills, and Joanna Scurr are with the Department of Sport and Exercise Sciences, University of Portsmouth, Portsmouth, UK. Address author correspondence to Alexandra Milligan at alex.milligan@port.ac.uk. [AUQ1]

Milligan, Mills, and Scurr

Multiplanar Breast Kinematics During Treadmill Running

http://dx.doi.org/10.1123/jab.2013-0121

\title{
Within-Participant Variance in Multiplanar Breast Kinematics During 5 km Treadmill Running
}

\author{
Alexandra Milligan, Chris Mills, and Joanna Scurr \\ University of Portsmouth
}

\begin{abstract}
More and more studies are emerging reporting breast kinematics. These studies rarely present effect sizes, power, and variance in the data. Important inferences are drawn from these data, including applications to product design, breast pain assessment, sports performance effects, and more. The aim of the study was to explore the within-participant variance in breast kinematic data during a $5 \mathrm{~km}$ run. Multiplanar breast kinematics and within-participant variance, defined by the coefficient of variation, for 10 female participants wearing a low- and high-level breast support were calculated during a 5 $\mathrm{km}$ run. Greater within-participant variance was reported in the high-level (mean $=15 \%$ ) breast support compared with the low-level (mean $=12 \%)$. Within-participant variance in breast kinematics did not change over the $5 \mathrm{~km}$ run. Differences in the magnitude of within-participant variance in breast kinematics were reported between directions of breast movement, with greater levels in the anteroposterior direction compared with mediolateral and vertical. It is important for the progression of this research area that the presence and sources of within-participant variance in breast kinematics are quantified and acknowledged, ensuring that the margin for meaningful differences can be reported.
\end{abstract}

Keywords: breast motion, sports bra, breast support, coefficient of variance

All tests and measurements are attended by error, ${ }^{1,2}$ and quantification of this error is required to conduct and interpret results within research. ${ }^{1,3}$ The following sources of error constitute total error in a measurement: systematic bias and random error. Random error can occur due to inherent biological or technical (measurement tool) variance, ${ }^{1,4}$ and is usually larger than systematic bias. The researcher can do little to reduce the biological aspect of random error because of its source. ${ }^{1}$ However, technical error of a measurement tool can be reduced to a certain extent by standardization procedures and calibration processes. $^{3,4}$

There are different methods to quantify the sources of error in data. The coefficient of variation $\left(\mathrm{C}_{v}\right)$, which is the ratio of the standard deviation to the mean, and expressed as a percentage, ${ }^{1,3}$ enables comparisons of error in measurements between studies utilizing different tools and measurements, regardless of units or calibration procedures. ${ }^{3}$ Hopkins $^{3}$ suggests the quantification of within-participant variance is the most important measure to consider when examining total error, as it can affect the precision of estimates of change in the measured variable.
Researchers should aim to calculate and if necessary report these data to ensure these sources are monitored and do not mask important effects.

With no muscles within the breast to damp and reduce soft tissue oscillations, ${ }^{5}$ the breast may elicit nonuniform movement patterns, which may influence the magnitude of variance inherent within these data. One source of within-participant variance may be a result of biological changes to the composition of the breast tissues between testing sessions; it is documented that breast volume may increase and density of the tissues alter during the luteal phase (day 14-28) of the menstrual cycle due to hormonal shifts. ${ }^{6-8}$ The effect these changes may have on breast kinematics is currently unknown; however, in an attempt to minimize this, where possible, participants could be tested during the more stable follicular phase (day 5-15) of the menstrual cycle.

Within breast biomechanics research, a marker positioned directly over the nipple is used to quantify and represent global breast kinematics. ${ }^{8}$ The ability of a participant to apply the marker directly over the breast in the same position may be considered as a source of 
within-participant variance; however, the following precautions are taken to reduce this source of variance. The explanation of the procedure should be clear, ensuring a reliable application between conditions, ensure the participants have been fitted in each breast support condition, and finally, the marker is not reapplied if knocked within the same trial.

Another source of within-participant variance, which is difficult to overcome, is variance in running technique between gait cycles. The thorax has been identified as the "driving force" behind breast movement"; therefore, alterations in the kinematics of the thorax, other body segments, and gait parameters may impact upon the relative kinematics of the breast, which may be more evident during longer duration running as the performer tires.

Scurr and colleagues ${ }^{8,10}$ reported the within- and between-participant variance in resultant breast displacement over constant and incremental speed treadmill protocols, lasting two minutes in duration. These are the only data available on variance in breast kinematics, and therefore, variance in breast kinematics has not been quantified over prolonged running distances. Gait kinematics and the variability in these data have been reported to increase over activity duration. ${ }^{11-14}$ Alterations in running mechanics during a run may therefore influence the magnitude of variance in breast kinematics. Furthermore, within-participant variance has only been reported for resultant breast displacement; therefore no inferences can be made regarding the variance in multiplanar breast kinematics. Magnitudes of breast kinematics have been reported to differ between the three directions of movement ${ }^{15}$; therefore, it could be hypothesized that variance in breast kinematics may also differ between the directions of movement.

Magnitudes of breast velocity and acceleration have been reported during running, ${ }^{15}$ but the associated variances in these data have yet to be examined. The derivative calculation of velocity and acceleration may exhibit greater magnitudes of variance due to the magnification of any error in the relative coordinate data during the calculation (magnification of 20 times at the second derivative stage). ${ }^{16}$ Therefore, the magnitude of variance in velocity and acceleration should be considered and reported, since the magnitude of variance in these data may mask different effects between conditions and trials.

The aim of the current study is to explore the within-participant variance in multiplanar breast kinematics in two levels of breast support, during a $5 \mathrm{~km}$ treadmill run. Firstly, it is hypothesized that breast support level will have a significant effect on the magnitude of within-participant variance. Secondly, the magnitude of within-participant variance will be significantly different across the intervals of the $5 \mathrm{~km}$ run. Thirdly, the magnitude of within-participant variance in multiplanar breast kinematics will be significantly different between the three directions of movement.

\section{Methods}

Following institutional ethics approval, ten female volunteers (experienced treadmill and outdoor runners currently training for at least $30 \mathrm{~min}$, at least three times per week) participated in this study. Participants had not had any children, not experienced any surgical procedures to the breast, and were of a $34 \mathrm{D}$ or $32 \mathrm{DD}$ bra size. Participants had a mean (SD) age of 23 years (2 years), body mass $62.1 \mathrm{~kg}(5.4 \mathrm{~kg})$, and height $1.60 \mathrm{~m}(0.05 \mathrm{~m})$. Participants provided written informed consent to partake in this study. The participants' bra size was measured by a trained bra fitter employing the best fit criteria recommended by White and Scurr. ${ }^{17}$

\section{Procedures}

In a random order, two $5 \mathrm{~km}$ treadmill runs $(\mathrm{h} / \mathrm{p} / \mathrm{cosmos}$, Germany) were performed on separate days (up to 72 hours apart); once in a low level breast support (Marks and Spencer Plain underwired T-shirt bra) and once in a high level breast support (Shock Absorber B4490 Sports bra) (Figure 1). Participants wore the same footwear for both treadmill runs. Participants selected a comfortable running speed, which they maintained for both $5 \mathrm{~km}$ runs without any adjustments, the average speed across all participants was $9 \mathrm{~km} \cdot \mathrm{h}^{-1}\left(1 \mathrm{~km} \cdot \mathrm{h}^{-1}\right)$.

\section{$\backslash$ Insert Figures 1 and $2 \backslash$}

Three retro-reflective markers (12 $\mathrm{mm}$ diameter) were positioned on the following anatomical landmarks by the researcher; the suprasternal notch (STN), and the left and right anteroinferior aspect of the 10th ribs (Figure 2) to represent the thorax segment. ${ }^{9,10} \mathrm{~A}$ fourth marker was positioned on the bra, directly over the nipple by the participant to represent the breast. ${ }^{8,18}$ A fifth marker was positioned on the right heel by the researcher to determine gait cycles using the procedure outlined by Zeni. ${ }^{19}$

Three-dimensional coordinates of the markers were tracked by eight $200 \mathrm{~Hz}$ calibrated Oqus infrared cameras (Qualisys, Sweden) positioned around the treadmill. The accuracy and precision of the motion capture system was determined utilizing two markers on a rigid calibration wand (Qualisys, Sweden), with a known intermarker distance of $750.7 \mathrm{~mm}$. The accuracy of the system was defined as the difference between the known intermarker distance and the mean reported intermarker distance recorded over three $10 \mathrm{~s}$ trials. The precision of the motion capture system was defined as the mean (SD) of these three trials (Table 1). The mean accuracy of the system was measured at $0.4 \mathrm{~mm}$, and the precision of the system was measured at $0.2 \mathrm{~mm}$. [AUQ2]

\section{$\backslash$ Insert Table $1 \backslash$}

The anatomical markers were identified and threedimensional data reconstructed in the Qualisys Track Manager (QTM) Software. Cameras recorded the last $10 \mathrm{~s}$ of the initial two minutes of the run and $10 \mathrm{~s}$ at each 
kilometer interval of the $5 \mathrm{~km}$ run $(900 \mathrm{~m}, 1900 \mathrm{~m}, 2900$ $\mathrm{m}, 3900 \mathrm{~m}$, and $4900 \mathrm{~m})$. Three-dimensional coordinates for all markers were exported to Visual3D (C-Motion v4, Inc., Germantown, MD).

\section{Data Processing}

The global coordinate system (GCS) identified $\mathrm{x}^{\prime}$ as the line of progression on the treadmill (anteroposterior), $\mathrm{y}^{\prime}$ as mediolateral, and $\mathrm{z}^{\prime}$ as vertical (Figure 2). Threedimensional coordinate data were filtered using a fourthorder zero-phase shift Butterworth filter with a cut-off frequency of $13 \mathrm{~Hz}$, determined using a FFT (MatLab, MathWorks).

To establish relative breast kinematics, independent to the six degrees-of-freedom movement of the thorax, an orthogonal segment coordinate system (SCS) converted the absolute coordinates of the nipple to relative coordinates using a transformation matrix within Visual3D. The three non-collinear markers positioned on the thorax were used to define the SCS, with the anteroinferior rib markers defining the medial and lateral locations of the distal end of the segment and the STN as the proximal end. A virtual midpoint (Figure 2) was established between the medial and lateral end points of the distal segment which extended to the suprasternal notch (origin and proximal end of the SCS) creating the vertical axis $\left(z^{\prime \prime}\right)$. The reference frontal plane $\left(y^{\prime \prime}-z^{\prime \prime}\right)$ was then defined using the three markers, with vector $y^{\prime \prime}$ perpendicular to the $\mathrm{z}^{\prime \prime}$. Vector $\mathrm{x}^{\prime \prime}$ was directed posterior to anterior, and using the right-hand rule was perpendicular to $\mathrm{z}^{\prime \prime}$ and $\mathrm{y}^{\prime \prime}$. Relative minima positional coordinates for the right nipple marker were subtracted from maxima coordinates to calculate breast displacement $(\mathrm{mm})$ relative to the thorax in each direction, across the five gait cycles at each interval of the $5 \mathrm{~km}$ run. First (velocity, $\mathrm{m} \cdot \mathrm{s}^{-1}$ ) and second (acceleration, $\mathrm{m} \cdot \mathrm{s}^{-2}$ ) derivatives of breast displacement were calculated instantaneously for each sample $(0.005 \mathrm{~s})$ with peak values recorded for each gait cycle $(n=5)$.

Coefficient of variance $\left(\mathrm{C}_{v}\right)$, reported as a relative percentage ${ }^{3}$ quantified the within-participant variance in relative multiplanar breast kinematics. The withinparticipant variance was calculated for each participant, for five gait cycles, ${ }^{8-10,15}$ at each interval of the $5 \mathrm{~km}$ run, for the low and high level of breast support, and then averaged across participants $(n=10)$.

\section{Statistical Analysis}

All data were checked for normality (KolmogorovSmirnov and Shapiro-Wilk) and homogeneity of variance (Mauchly's test of sphericity), and parametric assumptions assumed where $P>.05$. Two-way repeatedmeasures ANOVAs with post hoc pairwise comparisons (with Bonferroni adjustment) were performed to assess the effect of support level and run duration (intervals) on the magnitude of within-participant variance in multiplanar breast kinematics. Wilcoxon signed-rank tests were performed to identify any significant differences between the magnitudes of within-participant variance across the multiple directions of movement in the kinematic data, within each support condition.

\section{Results}

Level of breast support did not affect the magnitude of within-participant variance in anteroposterior or mediolateral breast displacement. However, variance in vertical breast displacement was greater in the high-level breast support $\left(F_{(1)}=22.382, P=.001, \eta^{2}=.713,1-\beta=\right.$ .987) when compared with the low-level breast support at the second $(P=.023)$, third $(P=.006)$, and fourth kilometer $(P=.016)$ of the run, increasing by $8 \%, 7 \%$, and $6 \%$, respectively from the low to high level of breast support.

Within-participant variance of multiplanar breast displacement did not differ over the $5 \mathrm{~km}$ run. However, within-participant variance in multiplanar breast displacement was significantly greater in the anteroposterior direction when compared with the mediolateral direction in the low $(Z=-4.340, P=.001)$ and high $(Z=-3.001, P=.001)$ level breast support. In addition, the variance in the vertical breast displacement was significantly greater than the mediolateral displacement $(Z=-4.697, P=.001)$.

Breast support level affected the magnitude of within-participant variance in breast velocity in the vertical direction $\left(F_{(1)}=64.404, P<.001, \eta^{2}=.877,1-\beta\right.$ $=1.000)$, with greater magnitudes of within-participant variance reported in the high level support compared with the low level support during the second $(P=.003)$, third $(P<.001)$, and fourth $(P=.17)$ kilometer of the run, (increasing by $7 \%, 10 \%$, and $8 \%$, respectively).

Within-participant variance in mulitplanar breast velocity did not differ over the $5 \mathrm{~km}$ run. Within the low level of breast support, greater magnitudes of withinparticipant variance were reported in the anteroposterior direction than the mediolateral $(Z=-2.179, P=.029)$ and vertical $(Z=-4.741, P<.001)$. Within-participant variance in the mediolateral breast velocity was greater than the variance in the vertical velocity $(Z=-3.129, P=$ .002). Within the high breast support, the withinparticipant variance was greater in the anteroposterior direction compared with the mediolateral direction $(Z=-$ $2.168, P=.030)$, a difference in variance of $3 \%$.

Level of breast support affected the withinparticipant variance in breast acceleration the vertical direction $\left(F_{(1)}=18.701, P=.002, \eta^{2}=.675,1-\beta=.969\right)$, with greater variance reported in the high level of breast support compared with the low level breast support during the second $(P=.020)$, third $(P<.001)$, fourth $(P=.005)$ and fifth $(P=.050)$ kilometer intervals, increasing by $9 \%$, $10 \%, 11 \%$, and $8 \%$, respectively.

Within-participant variance in multiplanar breast acceleration did not differ over the $5 \mathrm{~km}$ run. Greater magnitudes of variance in within-participant breast acceleration was found in the anteroposterior $(Z=-5.956$, 
$P<.001)$ and mediolateral $(Z=-5.013, P<.001)$ direction when compared with the vertical direction.

\section{Discussion}

This is the first study to explore the magnitude of withinparticipant variance in multiplanar breast kinematics between breast support conditions, during prolonged treadmill running. Within-participant variance in breast displacement across all directions, over the $5 \mathrm{~km}$ run in the low and high breast support averaged $7 \%$ and $10 \%$, respectively. As these data were derived for breast velocity the average variance increased to $13 \%$ (low) and $14 \%$ (high), and increased again to $17 \%$ (low) and $21 \%$ (high) for acceleration. These within-participant variance values are equivalent to a total error in breast kinematics of $3 \mathrm{~mm}, 0.04 \mathrm{~m} \cdot \mathrm{s}^{-1}$, and $2.8 \mathrm{~m} \cdot \mathrm{s}^{-2}$ in the low level support and $3 \mathrm{~mm}, 0.03 \mathrm{~m} \cdot \mathrm{s}^{-1}$ and $1.7 \mathrm{~m} \cdot \mathrm{s}^{-1}$ in the high level support. The technical error of the motion capture system was defined by the accuracy and precision of the system, and was established as less than $1 \mathrm{~mm}$. Therefore, the majority of the reported variance is assumed to be comprised of biological variance and systematic bias. This suggests that within the current population, differences of $3 \mathrm{~mm}$ (or less) in breast displacement, $0.04 \mathrm{~m} \cdot \mathrm{s}^{-1}$ (or less) in velocity, and $2.8 \mathrm{~m} \cdot \mathrm{s}-2$ (or less) in acceleration within and between high and low breast support conditions may not be true differences and instead may be due to total error.

When examining the effect of breast support level on within-participant variance in multiplanar breast kinematics during the $5 \mathrm{~km}$ run, variance in vertical breast displacement, velocity and acceleration were greater in the high-level breast support when compared with the lowlevel breast support, which partially accepts hypothesis one. Due to the calculation of the coefficient of variance, the magnitude of the mean and standard deviation have the potential to either elevate or reduce the value reported, specifically when examining small magnitudes. This may explain why the variance of breast kinematics was significantly greater within the high-level breast support when reported as a coefficient of variance. The benefit of using this relative statistic is clear when comparing across data with different methodologies or absolute units; however, presenting the absolute variance as well as a variability statistic will ensure accurate interpretation within and across research studies.

An arbitrary boundary of $10 \%$ has been proposed and is frequently employed as an "acceptable" level of variance within sports science research studies; ${ }^{4}$ however, this value is by no means definitive, and many studies within sports science research do not employ this guideline as a boundary for acceptable variance. Prior knowledge and implementation of this arbitrary boundary may influence the reader's interpretation of results across studies, utilizing different methods and measurement tools. Therefore, it is imperative to also consider the total error in the original units of measurement.
Within-participant variance of relative multiplanar breast kinematics was not reported to differ over the $5 \mathrm{~km}$ run. With no differences across the kilometer intervals, it is suggested that the magnitude of variance is consistent across prolonged treadmill running $(5 \mathrm{~km})$. This finding rejects hypothesis two and suggests that variance could be examined within the first two minutes of data collection. The decision for further examination of variance in these data are ultimately left to the investigator.

The magnitude of within-participant variance in breast displacement within the current study (average of low and high, across all directions, over five gait cycles = $9 \%$ ) was substantially greater than the variance reported by Scurr and colleagues ${ }^{8}$ (bare-breasted, over five gait cycles $=1.3 \%$ ). Scurr et $\mathrm{al}^{8}$ applied a log transformation to their data, ${ }^{8}$ which is advocated when data do not follow normal distribution and demonstrates heteroscedascity. ${ }^{1,3,20}$ This type of transformation uniforms the variance to produce a homogenous data set; however, it should be acknowledged that many biological parameters do not follow equal distributions due to the inherent random biological error, making these data unsuitable for log transformation. For the efficacy of future research in this area and to enable conclusive findings to be reported, within-participant variance in breast kinematics should be reported without log transformation. Moreover, future research on larger cohorts of participants, across multiple breast sizes, should establish generalized boundaries of error.

Separating breast kinematics data into individual directions of movement enables an understanding of which direction has larger magnitudes of variance. Anteroposterior and mediolateral breast kinematics frequently demonstrated greater within-participant variance than the vertical breast kinematics. This indicates more sporadic breast movement patterns in these directions, accepting hypothesis three. This may be explained by the bra's ability to reduce the vertical component of breast kinematics more effectively than the anteroposterior and mediolateral components. Previous publications within breast biomechanics have made recommendations for the design and structure of an effective breast support based upon data which focused only on the vertical component of breast kinematics. ${ }^{21,22}$ Therefore, the breast kinematics in the anteroposterior and mediolateral directions may be less uniform, creating greater variance in these data.

In conclusion, firstly, it was found that withinparticipant variance in breast kinematics is affected by breast support level, with greater variance reported in the high-level (15\%) compared with the low-level breast support $(12 \%)$. Secondly, it was reported that withinparticipant variance in breast kinematics remained constant over a $5 \mathrm{~km}$ treadmill run. Thirdly, the withinparticipant variance in anteroposterior breast kinematics was greater than the mediolateral and vertical. It is important for the progression of this research area that the presence of within-participant variance in breast kinematics is identified and quantified, and the source of 
this variance is acknowledged, ensuring that the margin for meaningful differences can be reported.

\section{References}

1. Batterham AM, George KP. Reliability in evidence-based clinical practice: a primer for allied health professionals. Phys Ther Sport. 2003;4:122-128. doi:10.1016/S1466-853X(03)00076-2

2. Payton CJ, Bartlett RM. Biomechanical evaluation of movement in sport and exercise: The British Association of Sport and Exercise Guidelines. Oxford, Abingdon: Routledge; 2008:129-152.

3. Hopkins WG. Measures of reliability in sports medicine and science. Sports Med. 2000;30(1):1-15. PubMed doi:10.2165/00007256-200030010-00001

4. Atkinson G, Neville AM. Statistical methods for assessing measurement error (reliability) in variables relevant to sports medicine. Sports Med. 1998;26(4):217-238.

PubMed doi:10.2165/00007256-199826040-00002

5. Page K, Steele JR. Breast motion and sports brassiere design. Sports Med. 1999;27:205-211. doi:10.2165/00007256-199927040-00001

6. Warren R. Hormones and mammographic breast density. Euro. Menopause J. 2004;49:67-78. PubMed

7. White E, Velentags P, Madelson MT, et al. Variation in mammographic breast density by time in menstrual cycle among women aged 40-49 years. J Natl Cancer Inst. $\quad$ 1998;90(12):906-910. $\quad$ PubMed doi:10.1093/jnci/90.12.906

8. Scurr J, White J, Hedger W. Breast displacement in three dimensions during walking and running gait cycles. $J$ Appl Biomech. 2009;25:322-329. PubMed

9. Haake S, Scurr J. A dynamic model of the breast during exercise. Int. Sport. Eng. Ass. 2010;12(4):189-197. doi:10.1007/s12283-010-0046-Z

10. Scurr J, White J, Hedger W. Supported and unsupported breast displacement in three dimensions across treadmill activity levels. J Sports Sci. 2011;29(1):5561. PubMed doi:10.1080/02640414.2010.521944

11. Williams KR, Snow R, Agruss C. Changes in distance running kinematics with fatigue. Int $J$ Sport Biomech. 1991;7:138-162.

12. Kyröläinen H, Pullinen T, Candau R, Arela J, Huttenen $\mathrm{P}$, Komi PV. Effects of marathon running on running economy and kinematics. Eur J Appl Physiol. 2000;82:297-304. doi:10.1007/s004210000219

13. Hardin E, Van Den Bogert A, Hamill J. Kinematic adaptations during running: Effects of footwear, surface, and duration. Med Sci Sports Exerc. 2004;36(5):838-844. doi:10.1249/01.MSS.0000126605.65966.40

14. Yoshino K, Motoshige T, Araki T, Matsuoka K. Effect of prolonged free-walking fatigue on gait and physiological rhythm. J Biomech. 2004;37:12711280. PubMed

15. Scurr JC, White JL, Hedger W. The effect of breast support on the kinematics of the breast during the running gait cycle. J Sports Sci. 2010;29(1):55-61. PubMed doi:10.1080/02640414.2010.521944

16. Pezzack JC, Norman RW, Winter DA. An assessment of derivative determining techniques used for motion analysis. Technical note. J Biomech. 1977;10:377382. PubMed doi:10.1016/0021-9290(77)90010$\underline{0}$

17. White J, Scurr J. Evaluation of the bra fitting criteria for bra selection and fitting in the UK. Ergonomics. 2012;55(6):704-711. doi:10.1080/00140139.2011.647096

18. Starr C, Branson D, Shehab R, Farr C, Ownbey S, Swinney J. Biomechanical analysis of a prototype sports bra. J. Text. Appar. Tech. Manag. 2005;4:1-14.

19. Zeni JA, Jr, Richards JG, Higginson JS. Two simple methods for determining gait events during treadmill and over ground walking using kinematic data. Gait Posture. 2008;27:710-714. PubMed doi:10.1016/j.gaitpost.2007.07.007

20. Bland JM, Altman DG. Transforming data. BMJ. 1996;312:770. doi:10.1136/bmj.312.7033.770

PubMed

21. Starr C, Branson D, Shehab R, Farr C, Ownbey S, Swinney J. Biomechanical analysis of a prototype sports bra. Journal of Textiles and Apparel. Technology and Management. 2005;4:1-14.

22. Mason BR, Page K, Fallon K. An analysis of the movement and discomfort of the female breast during exercise and the effects of the breast support in three case studies. J Sci Med Sport. 1999;2:134-144. PubMed doi:10.1016/S1440-2440(99)80193-5 


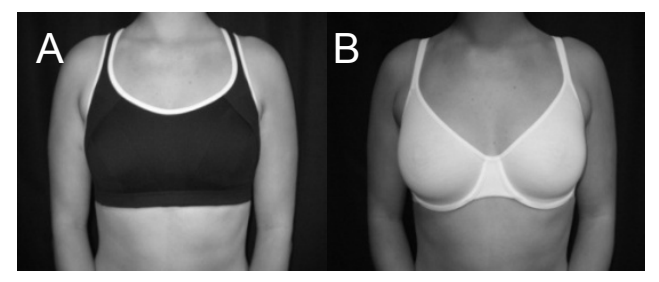

Figure 1 - (A) Sports bra: B4490, Shock Absorber level 4 support, made from 57\% polyester, 34\% polyamide, and $9 \%$ elastane. (B) Everyday bra: Marks and Spencer Seamfree Plain Under wired T-Shirt Bra, nonpadded, made from $88 \%$ polyamide and $22 \%$ elastane lycra. 


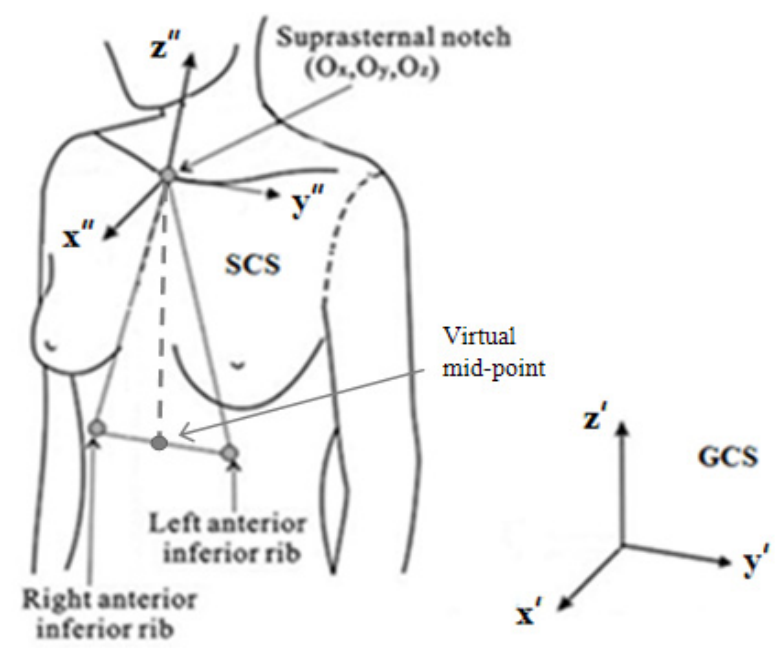

Figure 2 - Axis and coordinate system for the global coordinate system (GCS; $\left.x^{\prime}, y^{\prime}, z^{\prime}\right)$ and segment coordinate system (SCS; $\left.\mathrm{x}^{\prime \prime}, \mathrm{y}^{\prime \prime}, \mathrm{z}^{\prime \prime}\right)$. 
Table 1 Accuracy $(\mathrm{mm})$ and precision $(\mathrm{mm})$ of the motion capture system

\begin{tabular}{lcccc}
\hline & Trial 1 & Trial 2 & Trial 3 & Mean \\
\hline Intermarker Distance $(\mathrm{mm})$ & 750.2 & 750.2 & 750.4 & 750.3 \\
SD of Intermarker Distance (mm) (Precision) & 0.1 & 0.2 & 0.3 & 0.2 \\
Accuracy (mm) & 0.5 & 0.5 & 0.3 & 0.4 \\
\hline
\end{tabular}

Table 2 Mean \pm SD in multiplanar breast displacement $(\mathrm{mm})$ during the $5 \mathrm{~km}$ runs, in low and high breast support and the associated within-participant coefficient of variance $\left(\mathrm{C}_{v} \%\right)$

\begin{tabular}{|c|c|c|c|c|c|c|c|c|c|c|c|c|c|c|}
\hline & \multicolumn{14}{|c|}{ Intervals } \\
\hline & \multicolumn{2}{|c|}{$2 \min$} & \multicolumn{2}{|c|}{$1 \mathrm{~km}$} & \multicolumn{2}{|c|}{$2 \mathrm{~km}$} & \multicolumn{2}{|c|}{$3 \mathrm{~km}$} & \multicolumn{2}{|c|}{$4 \mathrm{~km}$} & \multicolumn{2}{|c|}{$5 \mathrm{~km}$} & \multicolumn{2}{|c|}{ Mean } \\
\hline & Low & High & Low & High & Low & High & Low & High & Low & High & Low & High & Low & High \\
\hline \multicolumn{15}{|l|}{ Displacement } \\
\hline $\mathrm{AP}(\mathrm{mm})$ & 34 & 24 & 36 & 27 & 38 & 27 & 38 & 28 & 37 & 26 & 37 & 27 & 36 & 27 \\
\hline $\mathrm{SD}(\mathrm{mm})$ & 3 & 6 & 3 & 6 & 4 & 6 & 5 & 6 & 5 & 6 & 7 & 7 & 5 & 6 \\
\hline Within $\left(\mathrm{C}_{v} \%\right)$ & 7 & 10 & 7 & 10 & 8 & 9 & 9 & 9 & 10 & 9 & 11 & 9 & 9 & 9 \\
\hline ML (mm) & 36 & 26 & 38 & 30 & 41 & 31 & 40 & 32 & 39 & 32 & 39 & 31 & 38 & 30 \\
\hline $\mathrm{SD}(\mathrm{mm})$ & 7 & 5 & 7 & 5 & 9 & 7 & 7 & 7 & 8 & 7 & 10 & 5 & 8 & 6 \\
\hline Within $\left(\mathrm{C}_{v} \%\right)$ & 6 & 8 & 5 & 6 & 6 & 7 & 6 & 7 & 6 & 6 & 6 & 6 & 6 & 7 \\
\hline $\mathrm{V}(\mathrm{mm})$ & 34 & 18 & 38 & 21 & 39 & 22 & 40 & 23 & 40 & 23 & 41 & 31 & 39 & 23 \\
\hline $\mathrm{SD}(\mathrm{mm})$ & 10 & 5 & 9 & 7 & 10 & 5 & 9 & 6 & 9 & 6 & 9 & 6 & 9 & 6 \\
\hline Within $\left(\mathrm{C}_{v} \%\right)$ & 8 & 11 & 7 & 11 & $6^{*}$ & $1 *$ & $6^{*}$ & $1 *$ & $4^{*}$ & $1 *$ & 10 & 12 & 7 & 13 \\
\hline
\end{tabular}

Note. AP, anteroposterior; ML, mediolateral; $\mathrm{V}$, vertical. ${ }^{*} P<.05$, significant difference between support conditions. 
Table 3 Mean \pm SD in multiplanar breast velocity $\left(\mathrm{m} \cdot \mathrm{s}^{-1}\right)$ during the $5 \mathrm{~km}$ run, in low and high breast support and the associated within-participant coefficient of variance $\left(C_{v} \%\right)$

\begin{tabular}{|c|c|c|c|c|c|c|c|c|c|c|c|c|c|c|}
\hline & \multicolumn{14}{|c|}{ Intervals } \\
\hline & \multicolumn{2}{|c|}{$2 \min$} & \multicolumn{2}{|c|}{$1 \mathrm{~km}$} & \multicolumn{2}{|c|}{$2 \mathrm{~km}$} & \multicolumn{2}{|c|}{$3 \mathrm{~km}$} & \multicolumn{2}{|c|}{$4 \mathrm{~km}$} & \multicolumn{2}{|c|}{$5 \mathrm{~km}$} & \multicolumn{2}{|c|}{ Mean } \\
\hline & Low & High & Low & High & Low & High & Low & High & Low & High & Low & High & Low & High \\
\hline \multicolumn{15}{|l|}{ Velocity } \\
\hline $\operatorname{AP}\left(\mathrm{m} \cdot \mathrm{s}^{-1}\right)$ & 0.28 & 0.18 & 0.29 & 0.19 & 0.36 & 0.20 & 0.32 & 0.21 & 0.34 & 0.19 & 0.32 & 0.20 & 0.32 & 0.20 \\
\hline $\mathrm{SD}\left(\mathrm{m} \cdot \mathrm{s}^{-1}\right)$ & 0.07 & 0.06 & 0.05 & 0.05 & 0.08 & 0.06 & 0.08 & 0.06 & 0.09 & 0.04 & 0.08 & 0.06 & 0.08 & 0.06 \\
\hline Within $\left(\mathrm{C}_{v} \%\right)$ & 19 & 16 & 17 & 14 & 12 & 15 & 14 & 13 & 15 & 17 & 19 & 14 & 16 & 15 \\
\hline $\operatorname{ML}\left(\mathrm{m} \cdot \mathrm{s}^{-1}\right)$ & 0.24 & 0.16 & 0.26 & 0.19 & 0.27 & 0.18 & 0.26 & 0.18 & 0.26 & 0.18 & 0.26 & 0.18 & 0.26 & 0.18 \\
\hline $\mathrm{SD}\left(\mathrm{m} \cdot \mathrm{s}^{-1}\right)$ & 0.04 & 0.04 & 0.04 & 0.05 & 0.06 & 0.04 & 0.04 & 0.04 & 0.04 & 0.05 & 0.05 & 0.05 & 0.05 & 0.05 \\
\hline Within $\left(\mathrm{C}_{v} \%\right)$ & 17 & 10 & 14 & 15 & 12 & 15 & 12 & 12 & 13 & 16 & 12 & 11 & 13 & 13 \\
\hline $\mathrm{V}\left(\mathrm{m} \cdot \mathrm{s}^{-1}\right)$ & 0.47 & 0.22 & 0.54 & 0.26 & 0.54 & 0.27 & 0.57 & 0.29 & 0.55 & 0.28 & 0.57 & 0.28 & 0.54 & 0.27 \\
\hline $\mathrm{SD}\left(\mathrm{m} \cdot \mathrm{s}^{-1}\right)$ & 0.15 & 0.10 & 0.17 & 0.11 & 0.19 & 0.10 & 0.18 & 0.11 & 0.18 & 0.11 & 0.16 & 0.10 & 0.17 & 0.11 \\
\hline Within $\left(\mathrm{C}_{v} \%\right)$ & 11 & 14 & 10 & 13 & $9 *$ & $16^{*}$ & $12^{*}$ & $22 *$ & $8^{*}$ & $16^{*}$ & 10 & 11 & 10 & 15 \\
\hline
\end{tabular}

Note. AP, anteroposterior, ML, mediolateral, $\mathrm{V}$, vertical. $* P<.05$, significant difference between support conditions. 
Table 4 Mean \pm SD in multiplanar breast acceleration $\left(\mathrm{m} \cdot \mathrm{s}^{-2}\right)$ during the $5 \mathrm{~km}$ run, in low and high breast support and the associated within-participant coefficient of variance $\left(C_{v} \%\right)$

\begin{tabular}{|c|c|c|c|c|c|c|c|c|c|c|c|c|c|c|}
\hline & \multicolumn{14}{|c|}{ Intervals } \\
\hline & \multicolumn{2}{|c|}{$2 \min$} & \multicolumn{2}{|c|}{$1 \mathrm{~km}$} & \multicolumn{2}{|c|}{$2 \mathrm{~km}$} & \multicolumn{2}{|c|}{$3 \mathrm{~km}$} & \multicolumn{2}{|c|}{$4 \mathrm{~km}$} & \multicolumn{2}{|c|}{$5 \mathrm{~km}$} & \multicolumn{2}{|c|}{ Mean } \\
\hline & Low & High & Low & High & Low & High & Low & High & Low & High & Low & High & Low & High \\
\hline Acceleration & & & & & & & & & & & & & & \\
\hline $\mathrm{AP}\left(\mathrm{m} \cdot \mathrm{s}^{-2}\right)$ & 13.9 & 7.0 & 13.9 & 7.0 & 16.3 & 8.7 & 14.2 & 7.6 & 15.4 & 7.9 & 14.4 & 7.9 & 14.7 & 7.8 \\
\hline $\mathrm{SD}\left(\mathrm{m} \cdot \mathrm{s}^{-2}\right)$ & 5.2 & 3.1 & 5.1 & 3.3 & 6.8 & 2.9 & 6.2 & 3.0 & 5.2 & 2.7 & 5.8 & 3.2 & 1.0 & 0.6 \\
\hline Within $\left(\mathrm{C}_{v} \%\right)$ & 23 & 20 & 18 & 20 & 16 & 30 & 19 & 21 & 21 & 20 & 21 & 23 & 20 & 22 \\
\hline $\operatorname{ML}\left(\mathrm{m} \cdot \mathrm{s}^{-2}\right)$ & 7.9 & 5.0 & 8.2 & 5.4 & 8.5 & 5.6 & 8.9 & 5.0 & 8.7 & 5.1 & 8.0 & 5.2 & 8.4 & 5.2 \\
\hline $\mathrm{SD}\left(\mathrm{m} \cdot \mathrm{s}^{-2}\right)$ & 2.1 & 1.3 & 2.1 & 1.7 & 2.5 & 1.7 & 2.8 & 1.3 & 2.4 & 1.8 & 2.0 & 1.4 & 0.4 & 0.2 \\
\hline Within $\left(\mathrm{C}_{v} \%\right)$ & 21 & 26 & 18 & 23 & 17 & 23 & 23 & 20 & 17 & 23 & 20 & 21 & 21 & 23 \\
\hline $\mathrm{V}\left(\mathrm{m} \cdot \mathrm{s}^{-2}\right)$ & 23.2 & 9.5 & 26.8 & 11.3 & 27.1 & 11.2 & 28.2 & 11.5 & 28.4 & 11.7 & 28.8 & 12.2 & 27.1 & 11.2 \\
\hline $\mathrm{SD}\left(\mathrm{m} \cdot \mathrm{s}^{-2}\right)$ & 6.9 & 5.3 & 7.5 & 6.0 & 8.8 & 5.0 & 8.3 & 5.1 & 8.0 & 2.2 & 6.5 & 4.6 & 2.1 & 0.9 \\
\hline Within $\left(\mathrm{C}_{v} \%\right)$ & 12 & 18 & 12 & 16 & $11^{*}$ & $20 *$ & $12 *$ & $22 *$ & $8^{*}$ & $19 *$ & $11^{*}$ & $19 *$ & 11 & 19 \\
\hline
\end{tabular}

Note. AP, anteroposterior, ML, mediolateral, $\mathrm{V}$, vertical. $* P<.05$, significant difference between support conditions. 


\section{Author Queries}

[AUQ1] It is my understanding that the conflict of interest in this case was pertinent only during the review stage, and thus I assume that the disclosure is not intended for the final publication. (Conflict of interest disclosure: Competing research group at the University of Wollongong.)

[AUQ2] Please provide "callouts" for the readers for Tables 2, 3, and 4. 